Chapter 11

\title{
Progress of Doppler Ultrasound System Design and Architecture
}

\author{
Baba Tatsuro \\ Additional information is available at the end of the chapter \\ http://dx.doi.org/10.5772/51508
}

\section{Introduction}

Evolution of electronic technology and semiconductor technology in recent years can realize a high-speed and high-quality signal-processing with low cost, low size, and low power consumption. Various signal-processing devices were born, and their performances are continuing developing. This article introduces the technical innovations and the effects of digital signal-processing in accordance with generations of the Doppler ultrasound system architecture. The diagnostic image of the carotid artery by a Doppler ultrasound system is shown in Fig. 1. The upside image is a tomogram called color flow mapping (CFM). A Doppler range gate is set up in the center of the blood vessel in the CFM. Bloodflow information on this position is displayed as the spectrum Doppler image in the downside. The horizontal axis is time, and the vertical axis is the flow velocity corresponding to Doppler shift frequency, and it expresses the time-change of velocity distribution of the bloodflow. The embedded technology of CFM and spectrum Doppler began from the composition of analog signalprocessing and primitive logical operation elements, and resulted to accumulator devices, PAL, various memories, and changed to FPGA, CPLD, ASIC, DSP, and CPU/GPU [1].

\section{Progress of Doppler signal-processing architecture}

\subsection{The 1st generation architecture (Fixed-point processing)}

Doppler signal-processing has developed selecting the most suitable realization method in all generations. Architecture of the 1980s is shown in Fig. 2. Analog signal-processing (dark-orange block in Fig. 2) occupied most in this architecture. Henceforth, this is called the 1st generation architecture. Since only fast Fourier transform (FFT) was the digital signal-processing, 
analog-digital converter (ADC) was arranged before FFT. In those days, the conversion speed of ADC was hundreds $\mathrm{kHz}$ in 12-16 bits. Since a complex butterfly-operation was required, FFT processing was realized by accumulators (TRW: 1010J) in the first stage. After a while, a fixed point DSP (Toshiba: DSP-T9508) was used from the second half of the 1980s.

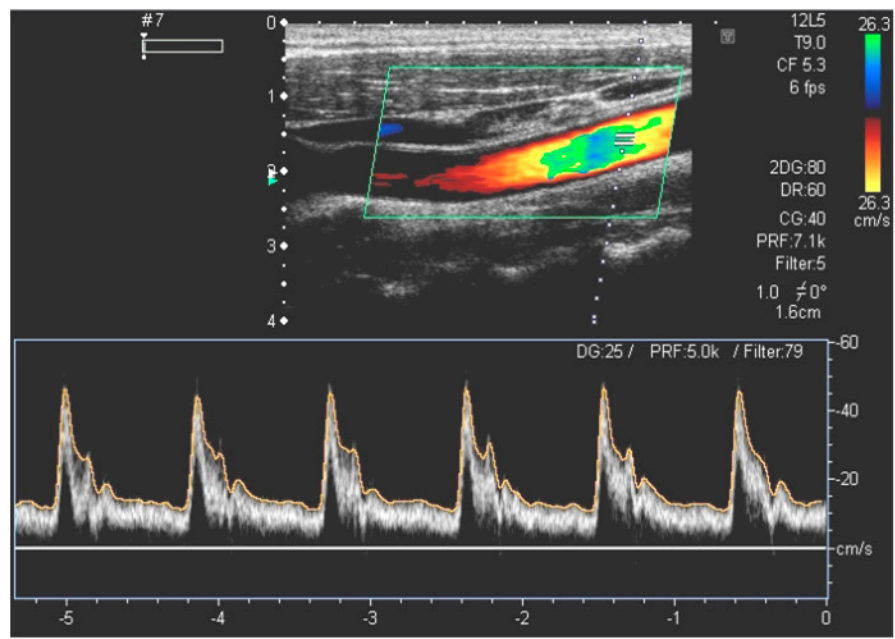

Figure 1. Diagnostic image of Doppler ultrasound system

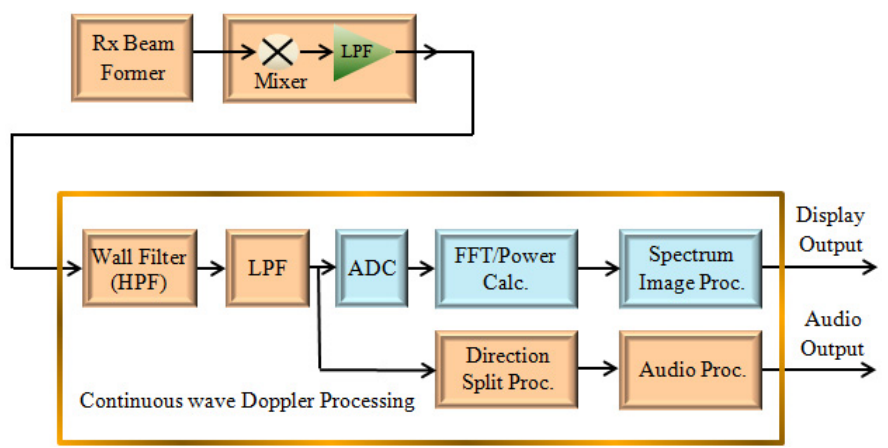

Figure 2. The 1st generation architecture

\subsection{The 2nd generation architecture (Floating point DSP and ASIC)}

The development of full digital system started early in the 1990s. The early digital architecture is shown in Fig. 3. Henceforth, this is called the 2nd generation architecture. An analog low-pass filter (LPF) was arranged after an analog high-pass filter (HPF) in the conventional analog signal-processing. Since it was difficult to realize a high-speed digital HPF (wall filter 
in Section 5) with low-cutoff, HPF and LPF were replaced. Furthermore, the LPF with high sampling frequency was divided into two subcomponents. The first stage LPF was realized by FPGAs (Altera: FPGA), and the next stage LPF and HPF were realized by floating point DSPs (NEC: $\mu$ PD77240A), respectively. Furthermore, FFT processing was realized by an ASIC (Toshiba: ASIC). However, as for Doppler audio processing (direction separation of complex signal, etc.), the conventional analog-circuit was used in consideration of cost-performance. Therefore, the digital filter output was converted into analog signal again by digital-analog convertoer (DAC), and was inputted into analog-circuit.

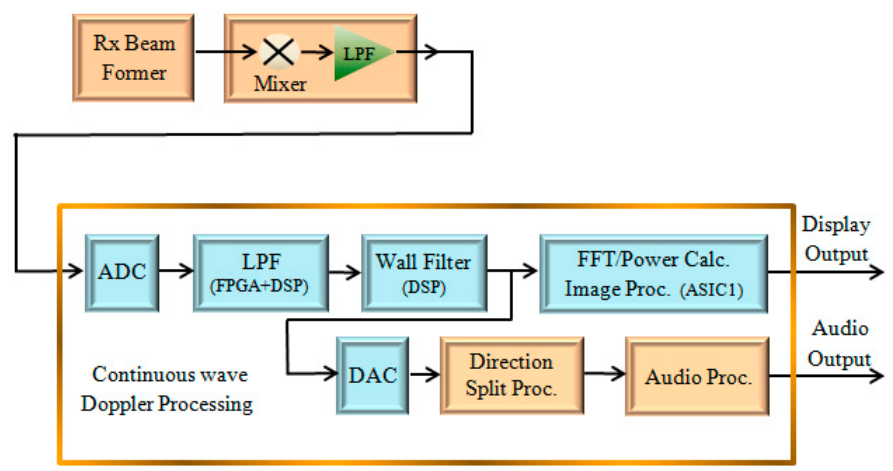

Figure 3. The 2nd generation architecture

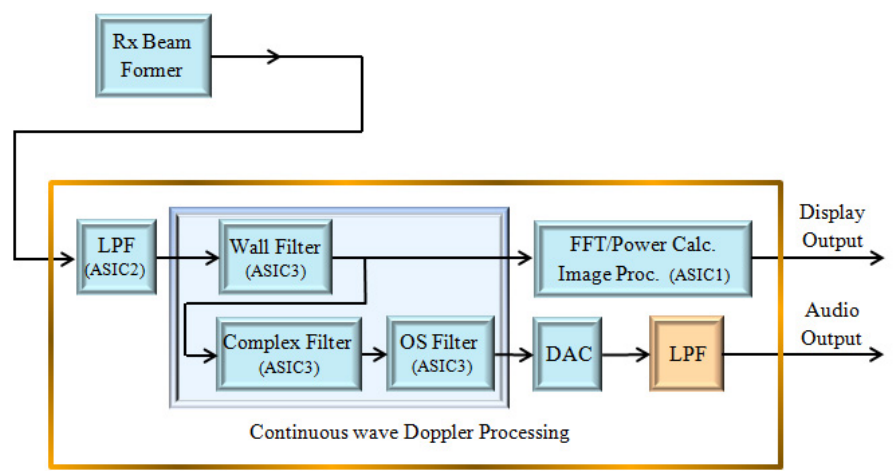

Figure 4. The 3rd generation architecture

\subsection{The 3rd generation architecture (Dynamic-range expansion by ASIC)}

In the second half of the 1990s, in order to merge CFM and spectrum Doppler, the development which reduces the size and cost of these systems started. Henceforth, this is called the 3rd generation architecture. In order to compare generations, only a spectrum Doppler portion is 
shown in Fig. 4. In the 3rd generation, since the system clock went up sharply, the floating point device was hard to use. CFM and spectrum Doppler were unified, and they were realized by five kinds of ASIC (Toshiba: fixed point ASIC). In this architecture, I adopted the newly developed digital complex IIR filter for the direction separation processing without using analog phase-shifters with heavy manual adjustments [2,3]. Furthermore, an oversampling filter and high-speed DAC (Analog Device: DAC) were used for the Doppler audio processing. This reduced the analog-circuit, such as high-order switched capacitor filters (SCF). The scale of these large-scale ASIC reached more than twice of typical CPU (Intel: Pentium processor) respectively, but the total cost of spectrum Doppler declined in $1 / 3$. Furthermore we were able to get the wide dynamic-range signal-processing which was difficult in analog processing. As a result, the sensitivity of bloodflow detection had improved and the diagnostic targets also spread to abdomen, surface blood vessels, and limbs etc.

\subsection{The 4th generation architecture (Reduction of circuits by large scale DSP)}

In the first stage of the 2000s I realized whole Doppler signal-processing using only one floating point DSP (TI: DSP TMS320C6701). The signal-processing block inside DSP is shown in Fig. 5. Henceforth this is called the 4th generation architecture. Since the clock frequency went up tens times compared with the 2nd generation floating point DSP, throughput improved sharply. Moreover, changes of the ultrasound system architecture contributed to downsizing. The interrupt cycle to DSP was changed into display frequency (Vsync: 50-75 $\mathrm{Hz}$ ) from ultrasonic pulse repetition frequency (PRF: 1-50 kHz). Although real-time performance was spoiled a little by forming packet processing, drastic reduction of circuit scales was realized.

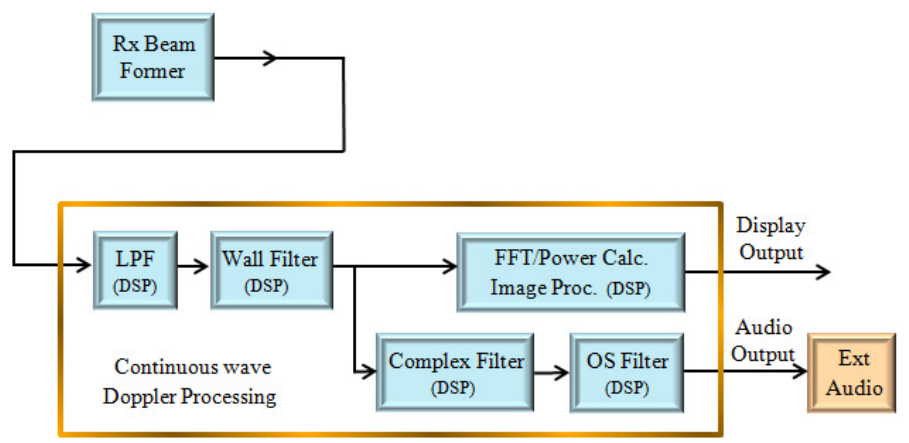

Figure 5. The 4th generation architecture

\subsection{The future architecture (Real-time analysis)}

Evolution of signal-processing had been influenced by the realization methods, like as from analog to digital, or from hardware to software. The size and cost of Doppler signal-processing were reduced day by day, and its performance (sensitivity or the response etc.) was also improved. It will be possible to gain more huge calculation power for signal-processing 
from now on. It will be also an important theme to investigate for what this power should be used. Now I am trying to apply this ability to automatic measurement and automatic diagnosis as an intellectual signal-processing [4-6]. Moreover, as another possibility, mounting the Doppler signal-processing on a Windows program is also tried. But problems, such as stability or response time, remain. In order to realize the real-time system which completes the processing within time, I think that the architecture based on DSP will remain.

\section{Considerations of digital technology}

As introduced in Section 2, the spectrum Doppler signal-processing architecture had changed from analog to digital in the first half of the 1990s. In this section, comparison of analog technology and digital technology and the innovations which digital technology brought about are introduced.

\subsection{Comparison of analog processing and digital processing}

As compared with analog processing, merits of digital processing are shown in Table 1. Digital processing realized quality improvement (reduction of variations), performance improvement (wide dynamic-range), and size reduction. Moreover, development efficiency was improved by the separation of analog power supply and digital power supply, and by the reduction of the noise in analog systems. Also the cost of digital-circuit had been improved in the 1990s more easily than analog-circuit.

\begin{tabular}{|c|c|c|}
\hline Items & Analog Processing & Digital Processing \\
\hline $\begin{array}{l}\text { Variation caused by } \\
\text { electrical parts }\end{array}$ & $\begin{array}{l}\text { Big. } \\
\text { (Expensive adjustment for analog-circuit) }\end{array}$ & Small. \\
\hline Tolerance to noise & $\begin{array}{l}\text { Noise countermeasure must be done to every } \\
\text { sub-block. } \\
\text { Limitation of dynamic-range }\end{array}$ & $\begin{array}{l}\text { Y Only ADC needs adjustment. } \\
\text { Wide dynamic-range is realizable. }\end{array}$ \\
\hline Kinds of power & $\begin{array}{l}\text { At least } 3 \text { kinds } \\
\text { The isolation between digital } \\
\text { Power supply and analog power supply is } \\
\text { needed. }\end{array}$ & $\begin{array}{l}\text { Only the digital power except for } \\
\text { ADC and DAC }\end{array}$ \\
\hline Cost performance & \multirow{2}{*}{$\begin{array}{l}\text { Before the 1990s, analog processing had } \\
\text { high C/P , but recently, it is low C/P compared } \\
\text {-with the digital processing. } \\
\text { Real time performance is good. }\end{array}$} & $\begin{array}{l}\text { Cost, size and power consumption } \\
\text { are improving substantially every } \\
\text { dyear. }\end{array}$ \\
\hline Throughput & & $\begin{array}{l}\text { Digital processing realizes the high-speed } \\
\text { and complex processing. }\end{array}$ \\
\hline
\end{tabular}

Table 1. Comparison of analog processing and digital processing 


\subsection{Time-spatial resolutions and $\mathrm{S} / \mathrm{N}$ ratio}

By development of digital technology, sampling frequency and pixel size are increasing in a digital camera and a digital audio field every year. When sampling frequency and pixel size increase, finer sampling becomes possible in time and space. The spatial-resolution and the time-resolution have improved recently, and a high-definition image and a high-fidelity audio can be enjoyed now. Moreover, the product performance that exceeds human vision and hearing is also improved. However, from the viewpoint of manufacturing cost, if the target performance to demand is filled, the present performance level may be enough. But the products which exceed this performance actually appeared one after another in the market. I consider this reason as follows. The present product level does not fill the dynamic-range of human vision and hearing. Since human sensitivity perceives physical quantity by logarithm, according to the surrounding environment, a wide dynamic-range is required. That is, I think that the commercial products of digital camera or digital audio have not reached the demand dynamic-range of luminosity or sound pressure yet [7-9].

Aside from improvement in spatial resolution or time resolution, another merit of digitization is a high $\mathrm{S} / \mathrm{N}$ ratio. A digital signal is sampled in a spacial axis and/or a time-axis. An ensemble mean processing can remove the noise which adjoins in space or time, so it can extract a low-frequency component with sufficient accuracy. An ensemble mean model, a signal level, a noise level, and expansion of $\mathrm{S} / \mathrm{N}$ ratio are shown in Fig. 6. When the ensemble number is set to $N$, a signal increases to $N$ times and a noise increases to $\sqrt{N}$ times so an $\mathrm{S} / \mathrm{N}$ ratio is expanded to $\sqrt{N}$ times $[10,11]$. Bandwidth restriction filters (HPF, LPF, BPF etc.) has the same effect of the ensemble mean processing. In digital processing designs, we should be cautious of an internal dynamic-range and an output noise level. In analog system, since the dynamic-range was narrow, a wide dynamic-range signal was not processed faithfully. The artifact caused by saturation or quantization occurred in the intermediate processes, so the sufficient sensitivity of bloodflow detection was not obtained. After the 3rd generation architecture, as the wide dynamic-range signal-processing was realized, the big improvement in bloodflow diagnosis was brought about.

\subsection{Hardware reduction by over-sampling}

The design concept of the compact disk (CD) which started in the 1980s was that the stereo digital signal (44 kHz sampling) was changed into the stereo analog signals (maintaining 20 $\mathrm{kHz}$ bandwidth). For this purpose, the steep analog filter (at least 7th-order) which rejects harmonics after AD conversion output $(22 \mathrm{kHz})$ was required. Several years afterward, 4times over-sampling system (about $170 \mathrm{kHz}$ ) appeared. It was realized by a digital moving average filter (sampling-frequency: about $170 \mathrm{kHz}$, cutoff frequency: $20 \mathrm{kHz}$, cutoff property: loose), a 4 times DA conversion, and a simple analog filter (cutoff frequency: $20 \mathrm{kHz}$, cutoff property: about 2nd-order). Since this system had many merits (reduction of cost and size, improvement of $\mathrm{S} / \mathrm{N}$ ratio etc.), it became mainstream [12].

The third generation architecture of Doppler ultrasound system was designed based on this over-sampling concept. In Doppler audio processing, unlike CD, a sampling frequency 
changes widely ( $1 \mathrm{kHz}$ to $50 \mathrm{kHz}$ ). Therefore, the cutoff frequency of digital filter before DA conversion had to be variable. The change range of sampling frequency is the same as not only Doppler audio processing but also digital filters of the 2 nd generation. The effect of the over-sampling processing is shown in Fig. 7. Fig. 7(a) shows the sampling characteristic of the sampling frequency $f s$, and harmonic (a side lobe of $-14 \mathrm{~dB}$ ) is mixed because of a simple over-sampling (hold characteristic). In order to remove these harmonics and to keep the required dynamic-range in required bandwidth, the filter with suitable bandwidth property (broken line) is required. In the case of Fig. $7(b)$, since $f_{S} / B W$ is small, a high-order filter is required. But in the case of Fig. 7(c), since $f_{s} / B W$ is large, a low-order filter is also fully realizable. The sampling frequency of high-speed digital devices is going up now. Since $f_{S} / B W$ is expanded, both downsizing and high-performance are realized simultaneously [13].

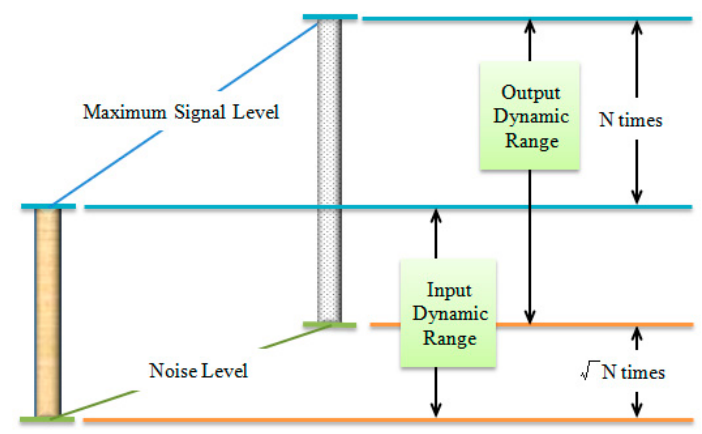

Figure 6. Principal of ensemble mean processing and $\mathrm{S} / \mathrm{N}$ ratio expansion
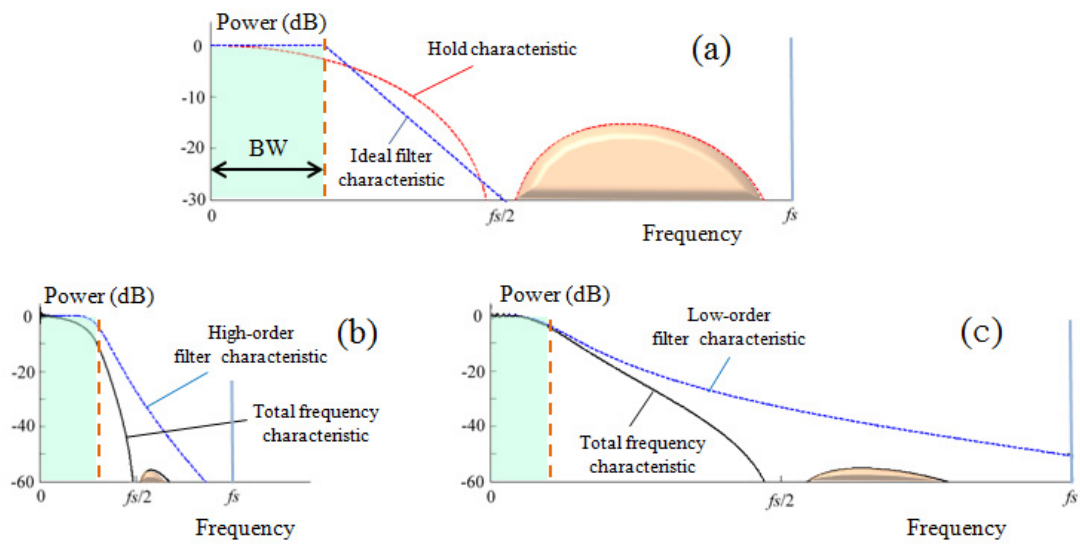

Figure 7. Over-sampling processing (a) Over-sampling model (b) Small fs/BW case (c) Large $f s / B W$ case 


\section{Cascade digital filter design}

The design tools for various digital filters, such as LPFs, HPFs, and BPFs, were popular and spread. However, in order to realize large-scale signal-processing combining various filters which have different sampling frequency, there are many points that should be taken into consideration, such as an aliasing and artifacts. In this section, two design examples of the cascade digital filter are introduced. One is a cascade BPF for continuous wave Doppler processing that has the down-sampling processing exceeding $10^{-4}$ or less. Another is a cascade LPF for Doppler audio processing that has the over-sampling processing exceeding $10^{3}$ or more.

\subsection{Continuous wave Doppler signal-processing}

In the Doppler signal-processing, an HPF is effective to extract weak bloodflow. There is a clutter signal which mixes unnecessary reflective ingredients from a blood vessel wall etc. By removing it at the entrance of frequency-analysis, the dynamic-range of FFT processing and audio processing can be held down, and also the signal-processing load can be reduced. High-order HPF array (bandwidth: several kHz, cutoff-frequency: several hundred to several thousand $\mathrm{Hz}$ ) had been used in the analog system. For digitizing this, the 2nd generation architecture was developed. The filter array for continuous-wave Doppler signal-processing is shown in Fig. 8.

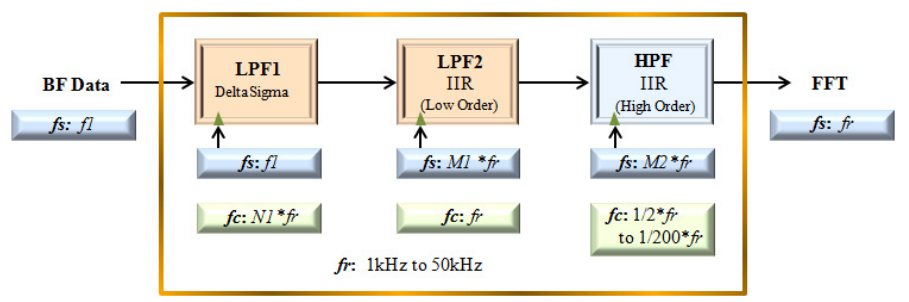

Figure 8. Digital filter design of continuous-wave Doppler signal-processing

In the conventional system, after an analog HPF, a bandwidth restriction was applied by the anti-alias LPF before ADC. To realize the digital HPF which had high-order and high sampling frequency was difficult in those days. For example, when a high-order IIR filter (several $\mathrm{MHz}$ and 2ch processing) was assumed, hundreds Mflps performance was required for calculation. Then, LPF was arranged before HPF in digitization. A high-speed and low-cutoff LPF was required. For example, the relative cutoff 1/1000 (that means several $\mathrm{kHz}$ bandwidth restrictions with tens of $\mathrm{MHz}$ sampling) was required. In order to prevent expansion of the tap-length (number of delay-registers) and bit-length of internal registers (inner dynamic-range), I chose the system which divided LPF into two steps and applied down-sampling. Since the output after quadrature detection was high-speed of tens $\mathrm{MHz}(f 1 \mathrm{~Hz}$ in Fig. $8)$, in the front part of LPF the delta-sigma LPF that had cutoff frequency N1*fr was adopted, and it was realized by FPGA. 
The latter part of LPF had re-sample frequency $M 1^{*} f r$, and it was realized by the single-precision floating point DSP (low-order LPF, cutoff-frequency: fr). An HPF was arranged after these two steps of LPFs. The HPF carried out scaling-processing to the LPF output by resampling frequency M2*fr. High-order and wide-range cutoff HPF processing (cutoff-frequency: $f r / 2$ to $f r / 200$ ) was realized by the double-precision floating point DSP. By this architecture, the required bandwidth restrictions and dynamic-range could be realized even in the continuous-wave Doppler processing which had heavy mixing of clatter artifacts. The frequency characteristic of the cascade digital filter (Fig. 8) is shown in Fig. 9. Actually a chirp waveform (0 to $40 \mathrm{kHz}$ ) was inputted into the cascade digital filter and its performance was checked by the spectrum Doppler image of the trial product. We can check the loose bandwidth restrictions near $\pm 6 \mathrm{kHz}(f r)$ in this figure.
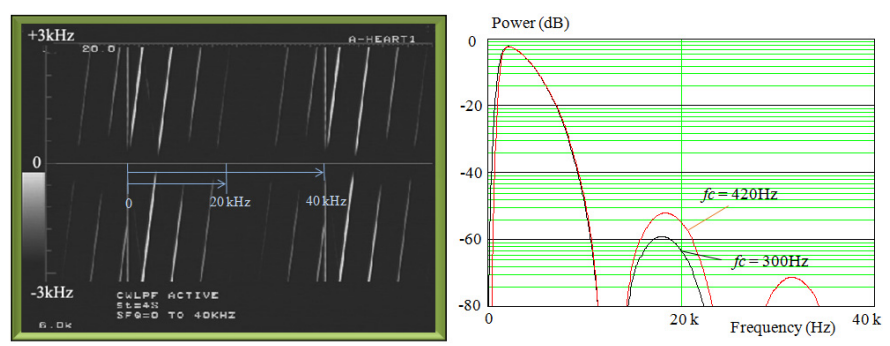

Figure 9. Frequency characteristics of continuous-wave Doppler filter of the 2nd generation architecture

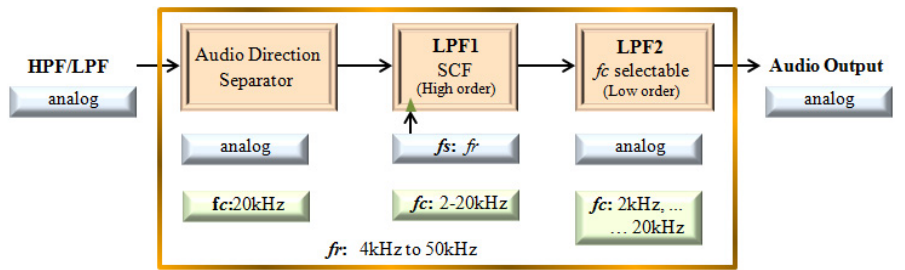

Figure 10. Analog Doppler audio system of the 2nd generation architecture

\subsection{Doppler audio signal-processing}

After quadrature detection, the Doppler audio system divides IQ signal into a forward component and a reverse component with a direction separation filter, and outputs them to the left and right stereo speakers. In the case of a pulse wave Doppler, since about $4 \mathrm{kHz}$ sampling frequency is interlocked with $f r$ (pulse repetition frequency), it is hard to hear bloodflow signal as it is (by the mixing of harmonics). In order to remove harmonics, it is required to realize the steep filter which has cutoff of $\mathrm{fr} / 2$ in consideration of audio bandwidth $(20 \mathrm{~Hz}$ to $20 \mathrm{kHz}$ ), and to reject unnecessary harmonics. The conventional analog filter architecture is shown in Fig. 10. In the Fig. 10, LPF1 had the steep cutoff characteristic by SCF after direc- 
tion separation processing. And LPF2 removed the harmonics generated in LPF1 (SCF noise). The $\mathrm{S} / \mathrm{N}$ ratio of SCF was $50 \mathrm{~dB}$ or less in audio-range, and sound quality was quite bad compared with the present system.

The Doppler audio filter of the 3rd generation is shown in Fig. 11. The signals of direction separation (complex BPF output) were oversampled by LPF1 whose sampling frequency $\left(M 3^{*} f s\right)$ was hundreds times larger than $f r$. And LPF1 had a loose bandwidth restriction by a moving average. In the following stage LPF2, scaling was applied by $f 2$ (same as the ADC clock frequency). LPF2 had the bandwidth restriction by the IIR filter (cutoff frequency: $f r / 2$ ). After AD conversion, in order to remove harmonics, loose bandwidth restriction was again applied by LPF3. This cascade filter processing could realize a high-quality Doppler audio (S/N ratio: more than $90 \mathrm{~dB}$ ). Moreover, since the oversampling frequency of LPF1 and the conversion frequency of ADC were set up more highly, the simple filter (lower-order) was used and drastic hardware reduction of LPF2 and LPF3 were realized [14].

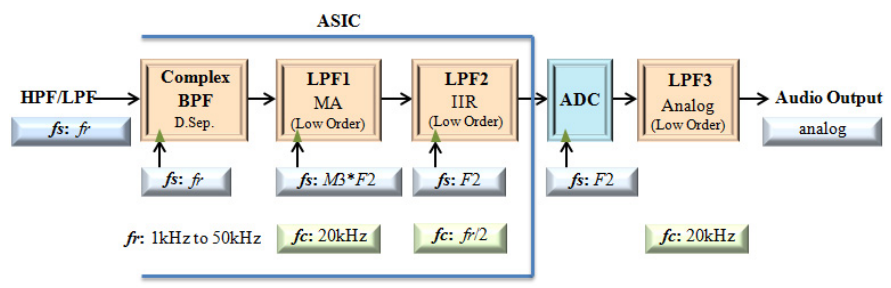

Figure 11. Digital Doppler audio system of the 3rd generation architecture

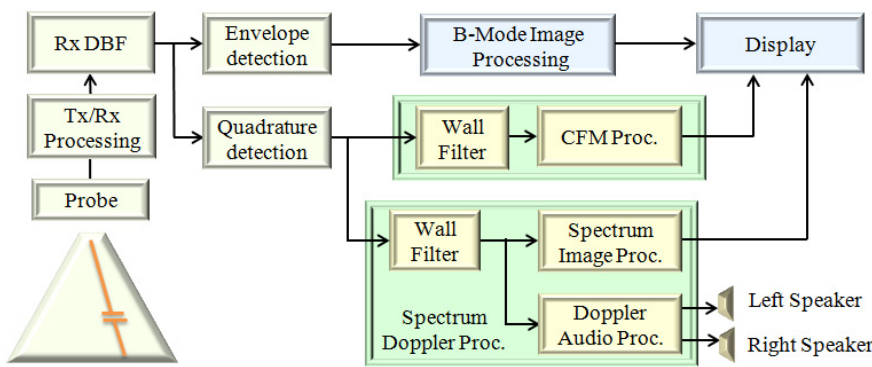

Figure 12. Wall filter arrangements of Doppler ultrasound system

\section{High-precision digital filter design}

Doppler ultrasound diagnostic method spread to many diagnostic fields, such as cardiac and abdomen. On the other hand, the improvement of bloodflow detection (sensitivity and velocity-range) had been desired for a long time. For this purpose, since it was required to 
separate weak bloodflow signal from high-power artifacts, like a blood vessel wall, the steep HPF (called a wall filter) had been arranged before frequency-analysis. With digitization, I investigated a new wall filter designs.

\subsection{Purpose of wall filter}

The locations of wall filters in Doppler ultrasound system are shown in Fig. 12. Quadrature detection outputs (IQ signal) are divided into CFM processing and the spectrum Doppler processing. In both CFM processing and the spectrum Doppler signal-processing, to save dynamic-range, wall filters are arranged before frequency analyses respectively [15].

Beam scanning methods and the display modes corresponding to them are shown in Fig. 13. Fig. 13(a) shows the scanning method of a B-mode echo image (tomogram), and it scans a beam from the right to the left. Fig. 13(b) shows the scanning method of spectrum Doppler, and it scans the same beam in a tomogram continuously. Fig. 13(c) shows the scanning method of CFM, and it scans a beam from the right to the left like Fig. 13(a), but the same beam is scanned twice or more. The sampling methods of Fig. 13(b) and Fig. 13(c) are shown in Fig. 14(a) and Fig. 14(b). Beam data is sampled by fr, and includes the information on the depth direction. As shown in Fig. 14(a), since the spectrum Doppler has a long time series signal, a detailed frequency analysis can be realized. On the other hand, as shown in Fig. 14(b), CFM has plurality data series (hundreds points) on the depth direction. Because CFM processing consists of a finite wall filter and a complex autocorrelation processing, the analysis data of CFM is same as sampling beam number (5 in the case of Fig. 14(b)), and is very small compared with that of the spectrum Doppler.

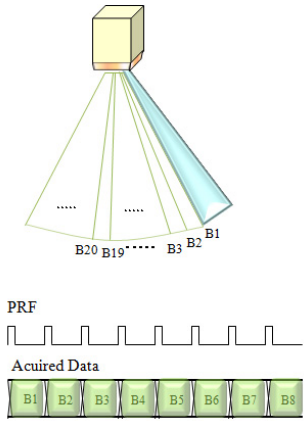

(a)

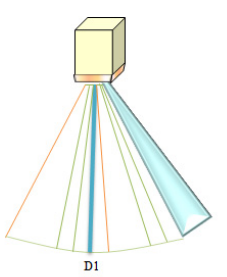

PRF

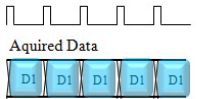

(b)
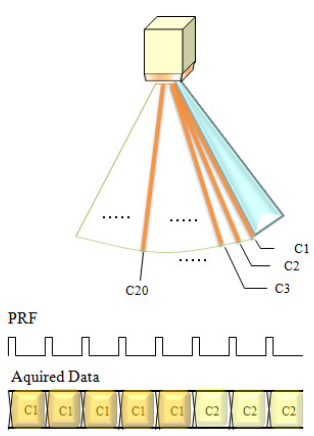

(c)

Figure 13. Ultrasound beam scan mode (a) B-mode (b) Spectrum Doppler (c) CFM

The clutter is the high-power and low-frequency component at the quadrature detection output. Compared with a bloodflow signal, it has high-power low-frequency component in abdomen (about $20 \mathrm{~dB}$ ) and heart (more than 40-60 dB). The quadrature detection outputs and wall filter outputs collected in the heart (left ventricle outflow) are shown in Fig. 15. The horizontal axes of Fig. 15(a) and Fig. 15(b) are time, and vertical axes are amplitude. The 
horizontal axis of Fig. 15(c) is frequency and a vertical axis is power. The wall filter has the 4th-order Butterworth characteristic with $200 \mathrm{~Hz}$ cutoff frequency. The power spectra of Fig. 15 (c) show that a big clatter component (20dB bigger) is removed. The wall filter is required high-order (steep) and low-cutoff characteristic [16].

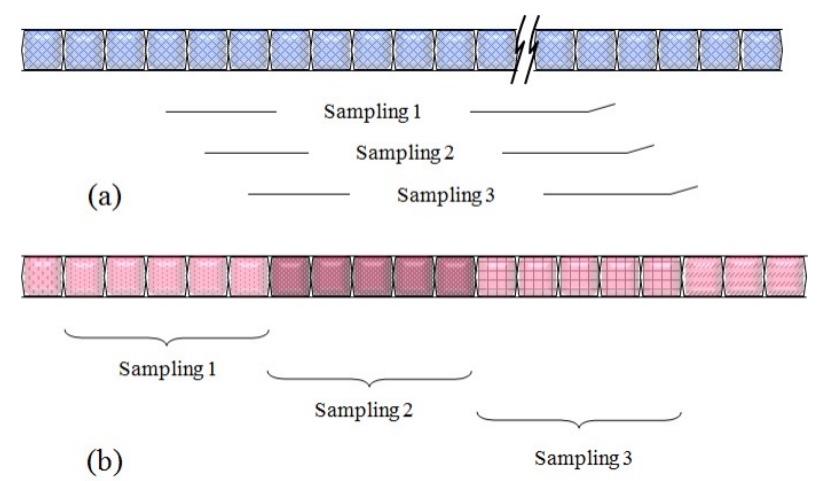

Figure 14. Sampling methods (a) Spectrum Doppler (b) CFM
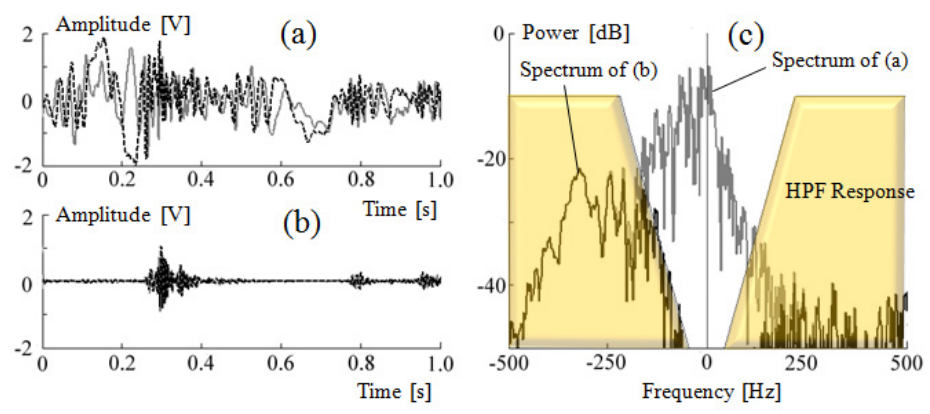

Figure 15. Effect of wall filter (a) Input signals of wall filter (b) Output signals of wall filter (c) Spectra of (a) and (b)

\subsection{Wall filter of CFM}

The high-order analog filter had been used for the wall filter of spectrum Doppler, and a finite digital filter had been used for the wall filter of CFM for a long time. The step responses of infinite impulse response (IIR) Butterworth filter in the case of changing cutoff and order are shown in Fig. 16. Fig. 16(a) shows the responses when order is changed at the relative cutoff frequency 1/16 (normalized by sampling frequency), and Fig. 16(b) shows the responses at the relative cutoff frequency 1/128. A transient response becomes long at the time of low-cutoff and high-order (steep). Relation between cutoff and order when the transient 
response is set to $-20 \mathrm{~dB}$ (10\% of step input amplitude) is shown in Fig. 16(c). Since performance of the wall filter with finite input is insufficient, the technology for reducing a transient response was required. The wall filter systems of CFM are shown in Fig. 17. In the finite impulse response (FIR) system of Fig. 17(a), if the number of delay registers $\mathrm{N}$ is small, sufficient performance cannot be obtained. So the IIR system of Fig. 17(b) became main-stream. However, unlike the wall filter of spectrum Doppler, the transient response of IIR filter has a serious influence to frequency-analysis. In order to solve this problem the adaptive filter which is consistuted by a time-variant FIR filter shown in Fig. 17(c) appeared recently.

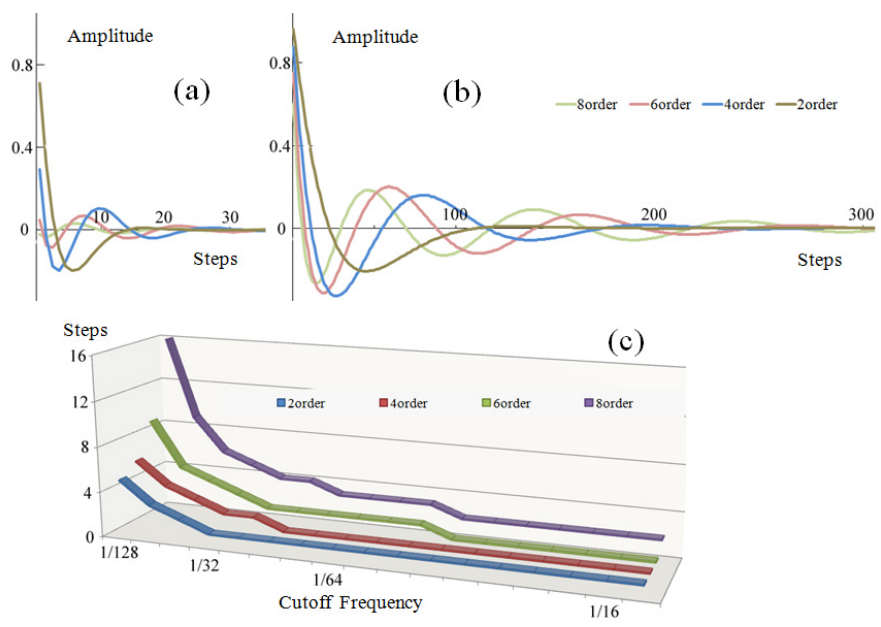

Figure 16. Step responses of HPF (a) Responses @ relative cutoff: 1/16 (b) Responses @ relative cutoff: 1/128 (c) Cutoff and transient response

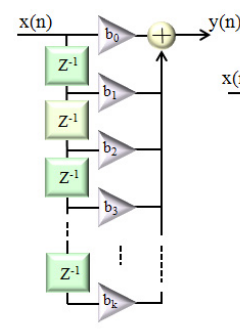

(a)

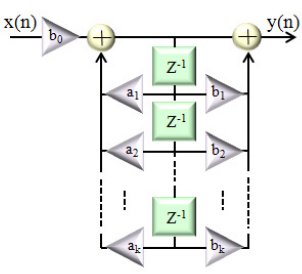

(b)

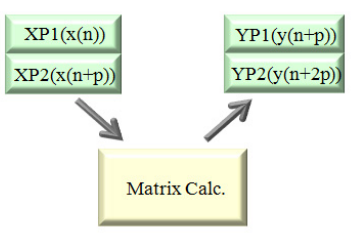

(c)

Figure 17. Wall filter systems of CFM (a) FIR filter (b) Biquad filter (c) Time valiant FIR filter

Since there is no feedback in the FIR system of Fig. 17(a), saturation does not occur easily, and the internal dynamic-range can be made small. However, since it is necessary to increase the 
number of taps in order to obtain low-cutoff, it is disadvantageous in a response and size. Biquad filter system of Fig. 17(b) has good response. However, since big internal dynamic-range is required at the time of low-cutoff, there are problems of a quantizing noise or a transient response. Compared with these, although time delay is given as a packet unit, a time-variant FIR filter system shown in Fig. 17(c) has many advantages. The filter response is calculated from packet data based on matrices. By progress of signal-processing device in recent years, the development of an adaptive filter based on the time-variant FIR system became also easy. A timevariant FIR filter with input $\mathrm{x}(\mathrm{n})$, output $\mathrm{y}(\mathrm{n})$, and the state variable $v(n)$ shown in Fig. 17(c) consists of the state equation and output equation in equation (1).

$$
\begin{gathered}
v(n+1)=F * v(n)+q * x 0(n) \\
y(n)=g^{T} * v(n)+d * x(n)
\end{gathered}
$$

The coefficients of an IIR filter (fig. 17(b)) are transposed into the matrix $F, q, g$ and $d$ in equation (2). The signal-processing equivalent to an IIR system can be realized by a timevariant FIR system.

$$
\begin{aligned}
F & =\left[\begin{array}{ccccc}
0 & 1 & 0 & \ldots & 0 \\
0 & 0 & 1 & \ldots & 0 \\
0 & 0 & 0 & \ldots & 1 \\
-a_{K}-a_{K-1} & -a_{K-2} & \ldots & -a_{1}
\end{array}\right] \quad q=\left[\begin{array}{c}
0 \\
0 \\
\ldots \\
0 \\
1
\end{array}\right] \\
g & =\left[\begin{array}{c}
b_{K}-b_{0} * a_{n} \\
b_{K-1}-b_{0} * a_{K-1} \\
\ldots \\
b_{1}-b_{0} * a_{1}
\end{array}\right] \quad d=b_{0}
\end{aligned}
$$

Thus, since the time-variant FIR system shown in Fig. 17(c) can solve both the problem of the internal dynamic-range and a transient response, it will develop as a new wall filter of CFM from now on.

\subsection{Wall filter of spectrum Doppler}

The step responses of 8 th-order Butterworth filter (4 cascade biquads, relative cutoff $1 / 128$ ) are shown in Fig. 18. Fig. 18(a) shows the step input $x(n)$ and the output $y(n)$. Since it is HPF, its output approaches DC with a damped oscillation. The responses of the inner registers for each stage $(Z 1(n), Z 3(n), Z 5(n)$ and $Z 7(n)$ in Fig. 20(b)) are shown in Fig. 18(b). Although $Z 3(n), Z 5(n)$ and $Z 7(n)$ are converged on DC with about tens times amplitude of an input, Z1(n) holds about 400 times amplitude of an input. Thus, when the HPF prevents saturation or keeps internal accuracy, wide dynamic-range of internal registers is required. The relation 
between cutoff and the dynamic-range (the bit-length of inner register) is shown in Fig. 19. In order to realize a high-precision digital filter, accuracy of operation registers and filter coefficients is important. I checked the minimum bit-length that was not influenced by quantizing noise. The responses of the fixed point 8th-order Butterworth filters were simulated. If quantizing noise is mixed, the unstable oscillation such as a limit cycle etc. will occur. I changed cutoff frequency and measured the limit of stability. As a result, in order to realize low cutoff, it turned out that sufficient mantissa-length of operation-registers and sufficient multiplication-coefficient length of multipliers were required. In fact, since cutoff frequency became about $1 / 200$ in the spectrum Doppler processing, a huge internal dynamic-range (about 200dB) was required.

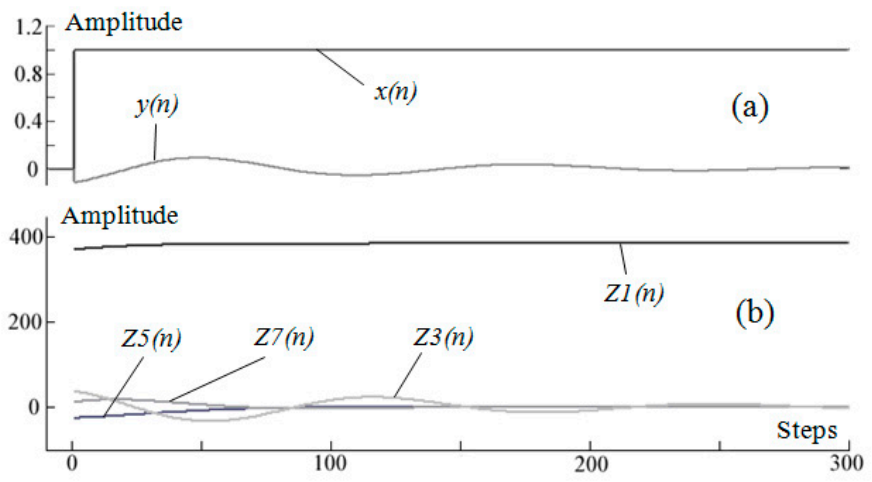

Figure 18. Step response of HPF and transit response (a) Input $x(n)$ and output $y(n)$ (b) Responses of internal resisters

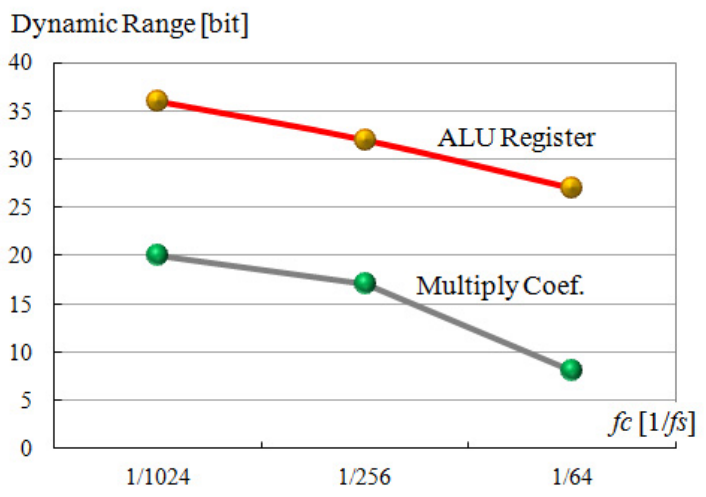

Figure 19. Dynamic-range (bit-length) and cutoff frequency of HPF 
The signal-processing architecture which reduces the internal dynamic-range of a digital filter was developed in the 2nd generation architecture. Simultaneously, the algorithm which reduces calculation in a real-time system was also investigated. The systems to realize 8thorder digital filter are shown in Fig. 20. Fig. 20(a) shows the loop system which makes the internal dynamic-range small with four delay-registers. Fig. 20(b) shows the loop biquad filter system with two delay-registers. The upper $Z^{-1}$ corresponds to Z1(n), Z3(n), Z5(n) and $Z 7(n)$ of Fig. 18(b). Fig. 20(c) shows the system with eight delay-registers in series. While the calculation cycle becomes small, the dynamic-range of internal registers becomes large.

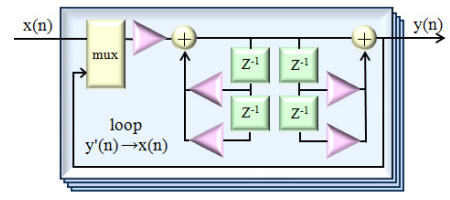

(a)

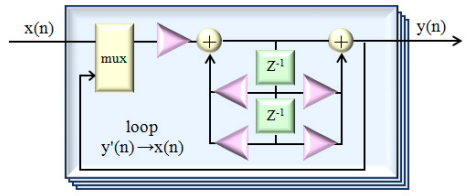

(b)

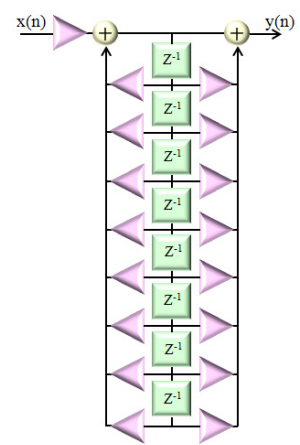

(c)

Figure 20. Wall filter systems of spectrum Doppler (a) Sysytem1: IIR+FIR loop system (b) System2: Biquad loop system (c) System3: Direct IIR system

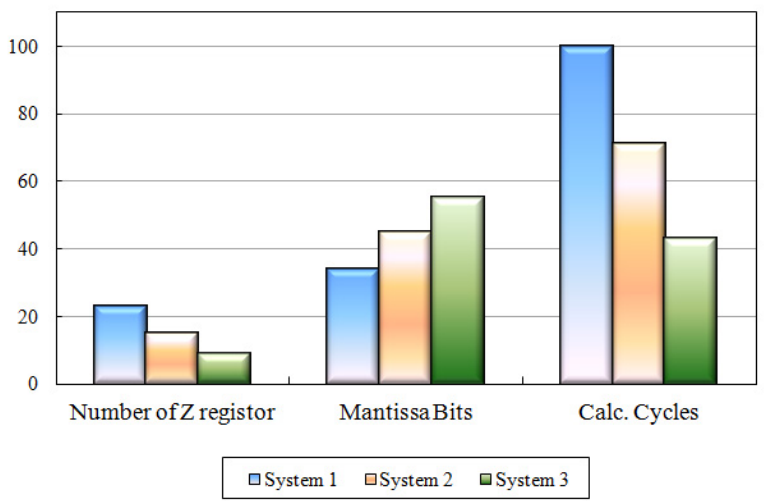

Figure 21. Benchmark based on floating point DSP ( $\mu$ PD77240)

Fig. 21 shows the evaluation of above systems. As benchmark condition, NEC $\mu$ PD77240 was used for floating point DSP. The 8th-order Butterworth HPF was chosen as benchmark proc- 
essing, and its relative cutoff was set to $1 / 256$. In the system 3 (equivalent to Fig. 20(c)), although both the number of double-precision registers and the operation cycles were small, internal bit-length became large. Since inner bit-length exceeded above 50 bits, even the double floating point arithmetic (mantissa-length: 48 bits) run short of accuracy, and was difficult to realize. In the system 1 (equivalent to Fig. 20(a)), many operation registers were required although internal bit-length was small. Since low-speed external memory access was required, its operation cycle increased. In the system 2 (equivalent to Fig. 20(b)), internal bit-length did not exceed the range of double-precision floating-point arithmetic, and an operation cycle was comparatively small. As mentioned above, the system 2 was judged the best system for mounting [17].

\section{Wide dynamic-range system design}

The dynamic-range of conventional system was insufficient for some clinical applications. In recent years, diagnostic ultrasound system was improved through the use of high-frequency electronics and integrated circuits. A new diagnostic method became effective by the higher dynamic-range system. However, the higher dynamic-range system also means more complicated gain control. It is possible to optimize the gain automatically through the use of ultrasound system parameters. This technology reduces the size of hardware and reduces the gain control range substantially.

\subsection{Signal-processing of ultrasound system}

Conventional ultrasound signal-processing is shown in Fig. 22. The transceiver processor (Tx/Rx Proc.) receives signals from the probe elements and the receive signals are amplified by the preamplifier. Gain compensation is applied to the signal to correct for range-distance attenuation (STC: sensitivity time control) and an analog gain correction for probe characteristics (frequency, sensitivity, etc) is applied. The signal is then sent to an ADC. After AD conversion the digital beam former (DBF) applies a delay pattern to the data to focus it and produce beam data. This data is processed by B-Mode Image Proc. and Doppler Image Proc., then displayed as a tomogram image and/or a spectrum Doppler image in the display processor (Display). The $\mathrm{S} / \mathrm{N}$ ratio is increased in the DBF as the number of channels (corresponds to transducer elements) to which delay calculations are applied increases [18, 19]. In Doppler signal-processing, quadrature detection (Mixer) is applied to the DBF output and a $\mathrm{BPF}$ provides band-limitation and clutter rejection. The result is a base-band Doppler signal. At this time, the $\mathrm{S} / \mathrm{N}$ ratio is sharply increased because of the band-limitation of the BPF. In the Doppler signal-processing, I apply range-gate integration (RG) across the range direction of the ROI. This also increases the $\mathrm{S} / \mathrm{N}$ ratio. In the case of continuous wave Doppler the dynamic-range is even larger. And the HPF applied to this data must be more sophisticated. The dynamic-range of the signal leaving the HPF is also much larger than in the pulse wave case, in the order of $100 \mathrm{~dB}$. After FFT the S/N ratio is greatly increased because of the butterfly integration. The dynamic-range of the signal is now very large and considerable gain adjustment and display compression must be done in order to display the data. 


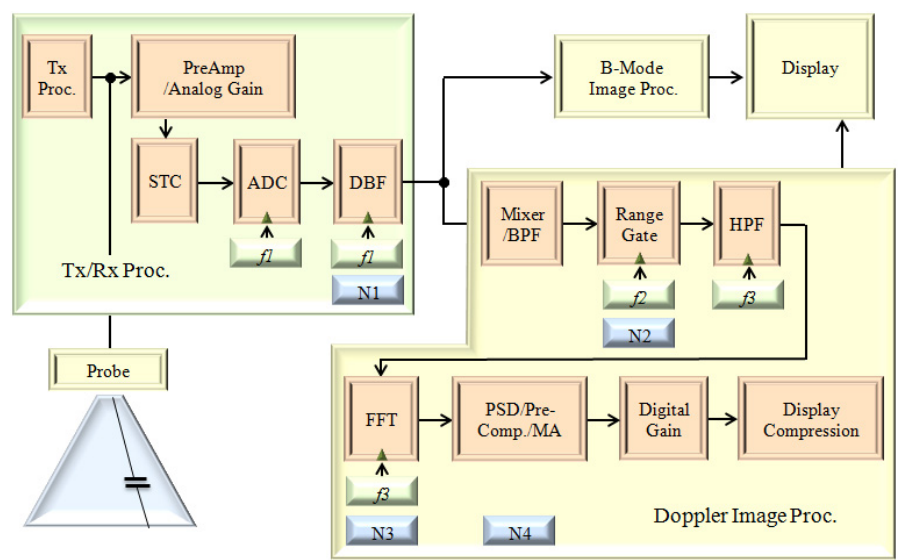

Figure 22. Ultrasound signal-processing system

It is also necessary to take into consideration that the dynamic-range is increased in Doppler signal-processing. In addition, mirror effect and/or quantization artifacts are introduced when performing automatic gain compensation. Although the beam data leaving the DBF has a frequency in the order of about $10-100 \mathrm{MHz}$, it is re-sampled at about 1-100 kHz. So the input dynamic-range of FFT increased by the band-limitation effect. Moreover the S/N ratio of FFT output is increased in a similar manner to that of ensemble mean processing.

\subsection{Purpose of gain adjustment}

Gain adjustment corrects for diagnostic target, bloodflow sensitivity, and the difference of user's skill. In addition to this, gain adjustment compensates for variation in other equipment parameters, such as the number of summing channel in DBF, apodization function, bandwidth of the $\mathrm{BPF}$, the integration length of the range-gate (RG), FFT number, window function, and the number of the shift addition of power spectrum according to sweep speed etc. The maximum signal level and a noise level change because of change of these equipment parameters. In order to realize highly sensitive Doppler bloodflow diagnosis without saturation, a system with wide dynamic-range must perform gain compensation according to all these parameters. Table 2 illustrates the rough estimation of the dynamic-range and $\mathrm{S} / \mathrm{N}$ ratio based on the virtual system [20].

The model of the signal-processing accompanied by expansion of the $\mathrm{S} / \mathrm{N}$ ratio is shown in Fig. 23(a). The noise level and the maximum signal level of the incoming signal are expanded by signal-processing. But the expansion of the noise level differs from the expansion of the maximum signal level, and the overall $\mathrm{S} / \mathrm{N}$ ratio is increased. Under optimal gain adjustment (range shown in light green in Fig. 23(a)) that there is no saturation of the maximum signal level and quantizing noise and signal are not mixed in the output. When gain adjustment is unsuitable (range shown in light pink in Fig. 23(a)), mirror effect or quantization artifacts occur on the spectrum image due to saturation or omission. As for signal amplitude, 
when quantization accuracy is inadequate, quantizing noise mixes. The gain adjustment that takes into consideration when detecting the weak Doppler signal around a system noise, and has sufficient quantizing margin is required. The influence of quantization is shown in Fig. 23(b). It is a spectrum when inputting sinusoidal $\left(0.02^{*} f s\right)$ including white noise. The horizontal axis is time and the vertical axis is frequency normalized by a sampling frequency $f$ s. The quantizing level of an input range was changed every 2 seconds with $3,5,9,17$. It turns out that the harmonics components $(-20$ to $-30 \mathrm{~dB})$ by quantization has occurred near the frequency $-0.3^{*} f s,-0.2^{*} f s$, and $+0.25^{*} f s$. The mirror effect is an imaginal image symmetrically generated with a real image on both sides of a baseline. In the analog system, it is mainly caused by the phase error of quadrature detection, or the small gain difference between IQ signals. In a digital system, although these influences do not receive, a mirror effect generates them owing to saturation. As shown in Fig. 23(c), on both sides of $0 \mathrm{~Hz}$, a symmetrical mirror effect occurs in the spectrum image. Fig. 23 (c) is the spectrum image which raised the gain $6 \mathrm{~dB}$ at a time every 2 seconds to the sinusoidal input including white noise. In this figure FFT input dynamic-range is 16 bit. The horizontal axis is time and the vertical axis is the frequency that normalized by a sampling frequency fs. The mirror effect component $\left(-0.2^{*} f s\right)$ has occurred by saturation to an original signal component $\left(+0.2^{*} f s\right)$. So in conventional ultrasound design both the mirror artifact and quantization artifact are caused by insufficient system dynamic-range in Doppler signal-processing.

\begin{tabular}{|c|c|c|c|c|}
\hline Module & Cause & $\begin{array}{l}\text { Effect of D.R. } \\
\text { Increment (*1) }\end{array}$ & $\begin{array}{l}\text { Conventional System } \\
\text { Fig. } 24(\mathrm{a})(\mathrm{dB})\end{array}$ & $\begin{array}{l}\text { New System } \\
\text { Fig. } 24(b)(d B)\end{array}$ \\
\hline ADC output & (Analog Gain) & $50 \mathrm{~dB}$ & DR1 @f1 & DR1 @f1 \\
\hline DBF & $\begin{array}{l}\text { BeamSum } \\
\text { effect(N1 } \\
\text { channel) }\end{array}$ & $+50 \mathrm{~dB}$ & $D R 2=D R 1+20 \log (N 1)$ & $\mathrm{DR} 2 \_\mathrm{opt}=\mathrm{DR} 1+20 \log (\sqrt{ } / N 1)$ \\
\hline Mixer/BPF & $\begin{array}{l}\text { BandLimitation } \\
\text { effect }(f 1 / f 2)\end{array}$ & $+30 \mathrm{~dB}$ & $\begin{array}{l}\text { DR3=DR1+DR2 } \\
+20 \log (f 1 / f 2)\end{array}$ & $\begin{array}{l}\text { DR3_opt=DR1+DR2_opt } \\
+20 \log (\sqrt{ }(f 1 / f 2))\end{array}$ \\
\hline RG & $\begin{array}{l}\text { RG Integration } \\
\text { effect(N2 tap) }\end{array}$ & $+40 \mathrm{~dB}$ & $\begin{array}{l}D R 4=D R 1+D R 2+D R 3 \\
+20 \log (N 2)\end{array}$ & $\begin{array}{l}\text { DR4_opt=DR1+DR2_opt } \\
+ \text { DR3_opt } \\
+20 \log (\sqrt{ } N 2)\end{array}$ \\
\hline FFT & $\begin{array}{l}\text { FFT number and } \\
\text { window(N3 } \\
\text { sampling) }\end{array}$ & $+50 \mathrm{~dB}$ & $\begin{array}{l}\text { DR5 }=D R 1+D R 2+D R 3+D R 4 \\
+20 \log (N 3)\end{array}$ & 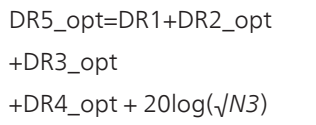 \\
\hline $\begin{array}{l}\text { PSD/Pre- } \\
\text { Compres. }\end{array}$ & $\begin{array}{l}\text { Power to } \\
\text { Amplitude }\end{array}$ & - & DR5 & DR5_opt \\
\hline MA & $\begin{array}{c}\text { Moving Average } \\
\text { effect(N4 } \\
\text { average) }\end{array}$ & $+10 \mathrm{~dB}$ & $\begin{array}{l}\mathrm{DR} 6=\mathrm{DR} 1+\mathrm{DR} 2+\mathrm{DR} 3+\mathrm{DR} 4 \\
+\mathrm{DR} 5 \\
+20 \log (N 4)\end{array}$ & $\begin{array}{l}\text { DR6_opt=DR1+DR2_opt } \\
\text { +DR3_opt } \\
+ \text { +DR4_opt+DR5_opt } \\
+20 \log (\sqrt{ } 4)\end{array}$ \\
\hline
\end{tabular}




\begin{tabular}{|c|c|c|c|c|}
\hline Module & Cause & $\begin{array}{l}\text { Effect of D.R. } \\
\text { Increment (*1) }\end{array}$ & $\begin{array}{c}\text { Conventional System } \\
\text { Fig. } 24(a)(d B)\end{array}$ & $\begin{array}{l}\text { New System } \\
\text { Fig. } 24(b)(d B)\end{array}$ \\
\hline \multirow{3}{*}{ Digital Gain } & $\begin{array}{l}\text { Digital Gain } \\
\text { Input }\end{array}$ & $\begin{array}{l}\text { D.R. before } \\
\text { Input }\end{array}$ & 230dB (DR6) & 140dB (DR6_opt) \\
\hline & $\begin{array}{l}\text { Digital Gain } \\
\text { Output }\end{array}$ & $\begin{array}{l}\text { D.R. after } \\
\text { Output }\end{array}$ & $\begin{array}{l}70 \mathrm{~dB} \\
(\mathrm{DR} 7)\end{array}$ & $\begin{array}{l}70 \mathrm{~dB} \\
(\mathrm{DR} 7)\end{array}$ \\
\hline & $\begin{array}{c}\text { Gain Control } \\
\text { Range }\end{array}$ & - & 160dB(DR6-DR7) & 70dB(DR6_opt - DR7) \\
\hline
\end{tabular}

(*1) This estimation is based on virtual model of the Doppler ultrasound system

Table 2. Comparison of inner dynamic-range and gain control range

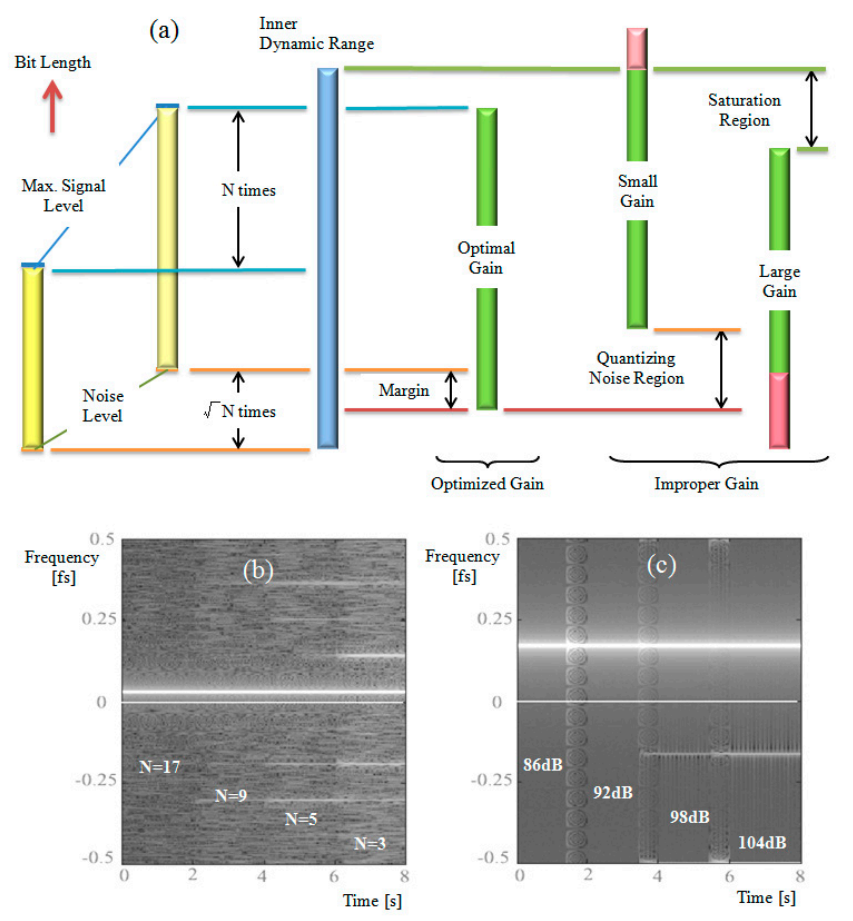

Figure 23. Artifacts caused by inadequate gain control (a) Problems of inadequate gain control (b) Artifacts caused by quantization (c) Artifacts caused by mirror effect.

\subsection{Wide dynamic-range design and its optimization}

Table 2 depicts the dynamic-range increment and the gain-control range increment of the conventional system and new system based on the signal-processing block diagram shown 
in Fig. 22. The DBF has the beam-summing effect of N1 channels. The Mixer/BPF has the band-limitation effect of $f 1 / f 2$. The RG has the integration effect of $N 2$ taps. The FFT has the integration effect (weighted by the window and operator) of N3. The PSD/Pre-Comp. just transfer the dimension (amplitude into power) using the square-root. The MA has the moving average effect of $\mathrm{N} 4$ columns according to the sweep speed of spectrum display. Fig. 24 shows the gain charts of the conventional system and new system based on Table 2. The gain chart of the conventional system which does not take the realization scale of hardware into consideration is shown in Fig. 24(a). This time, I developed the system that can reduce the gain-control range and can also reduce an internal dynamic-range. The automatic gain compensation according to the change-range of system parameters is realized for every subblock of Doppler signal-processing accompanied by improved $\mathrm{S} / \mathrm{N}$ ratio. Since the ranges of system parameters are known, the improvement of $\mathrm{S} / \mathrm{N}$ ratio, the maximum signal level, and the noise level are calculable. An internal dynamic-range scale and the gain adjustment range can be optimally designed for every sub-block. By connecting the partial optimal subblock in series and uniting it the internal dynamic-range of the system can be reduced so the system size and the total gain control range can both be sharply reduced. The internal S/N ratio is increased by $\sqrt{N}$. Then, supposing an input signal dynamic-range is DRin [dB], the range expansion equivalent to $20^{*} \log (\sqrt{N})$ [dB] will occur. Moreover, the internal dynamicrange DRproc $[\mathrm{dB}]$ which added more than the margin $\left(20^{*} \log (\sqrt{12})\right.$ to quantizing noise is roughly calculable using equation (3).

$$
\text { DRproc } \geq \text { DRin }+20 \cdot \log (\sqrt{N})+20 \cdot \log (\sqrt{12})
$$

When digitizing, the Least Significant Bit (LSB) must be rounded not truncated otherwise an error of $1 / 2^{*} \mathrm{LSB}$ will exist. The RMS value of the quantizing noise is equivalent to $1 / 2^{*} \mathrm{LSB} /$ $\sqrt{3}$. So an additional margin of $\sqrt{12}$ for dynamic-range must be maintained so rounding can be performed accurately. Although the internal dynamic-range DRproc is enough only in the automatic gain compensation with system parameters, it is necessary to consider a margin to the original gain adjustment that adjusts for diagnostic target and Doppler sensitivity variation at an internal dynamic-range. The gain chart of the sub-block signal-processing accompanied by range expansion is shown in Fig. 24(b). The gain-control range of the conventional system (corresponds to Fig. 24(a)) is DR6-DR7, and the gain-control range of the new system (corresponds to Fig. 24(b)) is DR6_opt-DR7. In the general Doppler signal-processing DR6 is above $200 \mathrm{~dB}$, and DR7 (the digital gain output) is display luminance range (about 70 $\mathrm{dB}$ ). So the gain-control range of conventional system should be more than $130 \mathrm{~dB}$. This is very large. On the other hand the DR6_opt of new system is smaller than DR6 about $100 \mathrm{~dB}$. I can reduce not only gain-control range but also the inner dynamic-ranges of sub-modules at the same time [21].

The effect of the automatic gain optimization using the new system was checked in a simulation of RG-integral processing. The spectrum images of the conventional system and the new system when changing RG-width are shown in Fig. 25(a) and Fig. 25(b). The horizontal 
axis is time and the vertical axis is the frequency normalized by a sampling frequency fs. The range-gate was adjusted from $1 \mathrm{~mm}$ to $4 \mathrm{~mm}$ to $16 \mathrm{~mm}$ in $1.8 \mathrm{~s}$ intervals. A sinusoidal signal including white noise was used as an input. In the conventional system of Fig. 25(a), the signal level and noise level increase with expanding RG-width. For this reason, the user should reducing gain manually when the RG-width is expanded. In the new system in Fig. 25(b), although the signal level will rise if RG-width is expanded, it turns out that a noise level does not change. As mentioned above, by Doppler automatic gain compensation, the input bit length of each signal-processing block could be made smaller, and also the gain adjustment range could be made small to necessary minimum.
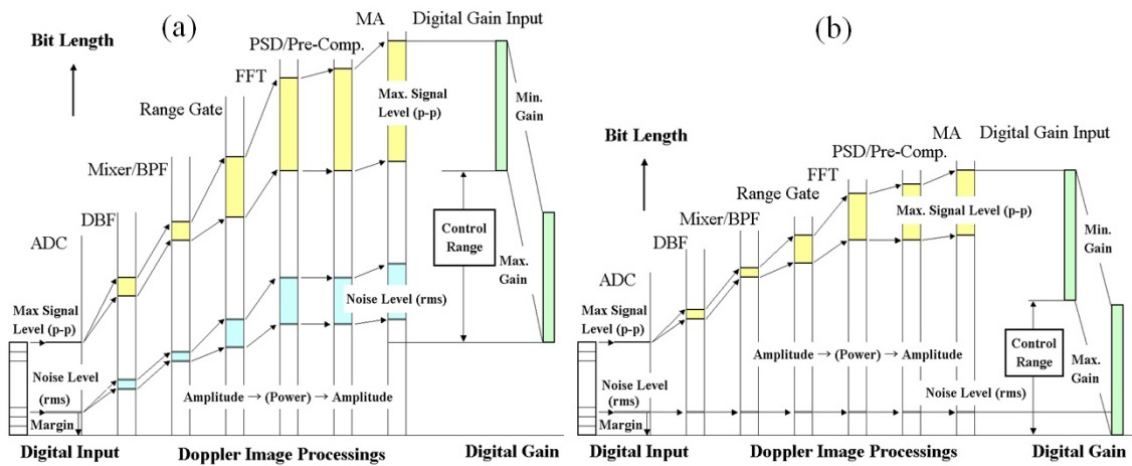

Figure 24. Comparison of gain control systems (a) Gain chart of conventional system (b) Gain chart of new system.
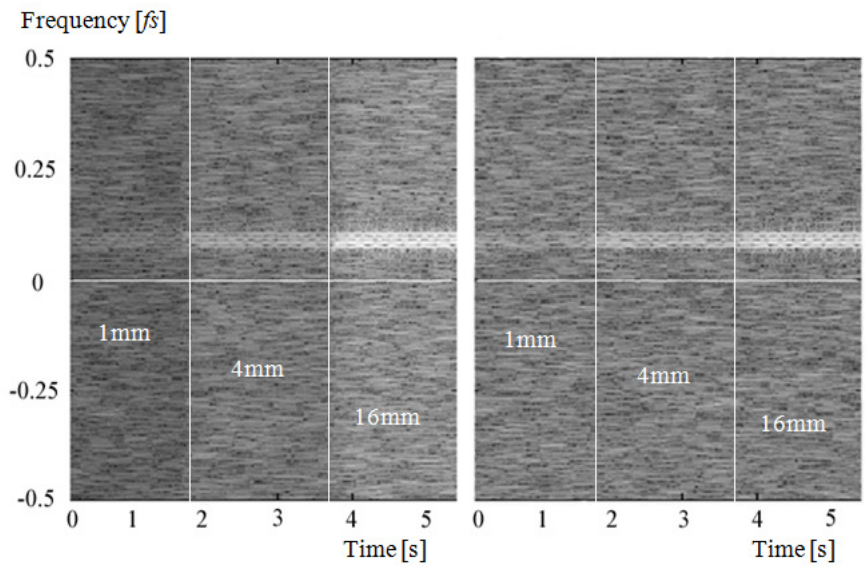

Figure 25. Effect of automatic gain compensation: example of RG integration process. (a) Conventional system (b) New system 


\section{Conclusion}

The technical innovations which digital signal-processing brought about and their results were introduced based on some examples of the Doppler ultrasound system architectures. Not only extensive improvement of cost, size, power consumption, and adjustment, but also the improvement of sensitivity and accuracy has been realized by digital technology. Although DSP is most suitable for real-time system at present, the system architecture will be mounted as software when the calculation power of CPU/GPU improves further. In the future we will be able to acquire huge calculation ability easily, and it will be possible to apply it to real-time automatic diagnostic technology etc. besides conventional signal-processing.

\section{Author details}

Baba Tatsuro

Address all correspondence to: EZD03014@nifty.ne.jp

Toshiba Medical Systems Corporation, Japan

\section{References}

[1] Baba, T. (2009). Progress of Doppler ultrasound system architecture and considerations: The problems caused by digital system and their solutions. Society of Signal Processing Applications and Technology of Japan, 12(1), 2-8.

[2] Baba, T. (2005). Investigation on direction split technique of Doppler ultrasound: Comparison of six kinds of Doppler audio processing. Society of Signal Processing Applications and Technology of Japan, 8(2), 14-20.

[3] Baba, T. (2007). Direction separation in Doppler audio of ultrasound diagnosis equipment: Signal processing for Doppler audio dealiasing. Acoustical Science and Technolo$g y, 28(3), 202-210$, DOI: ast.28.202.

[4] Baba, T. (2005). Research on Doppler ultrasound automatic heartbeat cycle detection: The investigation of heartbeat cycle detection from Doppler waveform using adaptive BPF. The Journal of the Acoustical Society of Japan, 61(11), 629-635.

[5] Baba, T. (2006). Velocity range tracking in Doppler diagnostic ultrasound systems: Range optimization using Doppler trace wave form histograms. The Journal of the Acoustical Society of Japan, 62(4), 327-331.

[6] Baba, T., Ohmae, N., \& Osuka, K. (2008). The Optimization of Ultrasound System Doppler Velocity Range using Hybrid Control. Transactions of the Society of Instrument and Control Engineers, 44(9), 760-765. 
[7] Ohashi, T. (2001). Recording of World Heritage on the High Definition Audio-visual Media: Documentation of History and Tradition. ITE Transactions on Media Technology and Applications, 55(1), 37-46.

[8] Yoshikawa, S. (2002). Present status of high definition audio. The Journal of the Acoustical Society of Japan, 58(4), 250-255.

[9] Misawa, T. (2004). The image sensor for digital cameras. Japanese Journal of Optics, 33(9), 544-549.

[10] The Physical Society of Japan. (1978). Physics experiment data processing by a computer, the 4th edition. Tokyo: SAIENSU-SHA Co., Ltd.

[11] Miyagawa, H. (1981). Digital Signal Processing The 9th edition. Tokyo: CORONA PUBLISHING Co., LTD.

[12] Nakajima, T. (1996). Compact disk reader, the 3rd edition. Tokyo: Ohmsha Ltd.

[13] Baba, T., \& Toshiba, Corp. (2008). Ultrasonic diagnostic equipment. Japanese Patent 4068208; Jan. 18.

[14] Baba, T., \& Toshiba, Corp. (2006). Ultrasonic diagnostic equipment. Japanese Patent 3746113, Feb. 15.

[15] Jensen, JA. (1996). Estimation of Blood Velocities Using Ultrasound: A Signal Processing Approach:. Cambridge University Press, 0-521-46484.

[16] Baba, T. (2008). Evaluation of Post Wall Filter for Doppler Ultrasound Systems. Acoustical Imaging, 29-133, 978-1-40208-822-3.

[17] Baba, T. (2006). Investigation of wall filters in Doppler ultrasound system. Society of Signal Processing Applications and Technology of Japan, 9(2), 14-19.

[18] Kozak, M., \& Karaman, M. (2001). Digital Phased Array Beamforming Using SingleBit Delta-Sigma Conversion with Non-Uniform Oversampling. IEEE Transactions on UFFC, 48(4), 922-931, DOI: 10.1109/58.935709.

[19] Engelberg, S. (2006). Implementing a $\Delta \sum$ DAC in Fixed Point Arithmetic. IEEE Signal Processing Magazine:, DOI:10.1109/SP-M.2006.248716, 66-69.

[20] Baba, T., \& Toshiba, Corp. (2005). Ultrasonic diagnostic equipment. Japanese Patent 3663206.

[21] Baba, T. (2009). Investigation of gain optimization technique in Doppler ultrasound system. Acoustical Science and Technology, 30(2), 61-71, DOI: ast.30.67. 